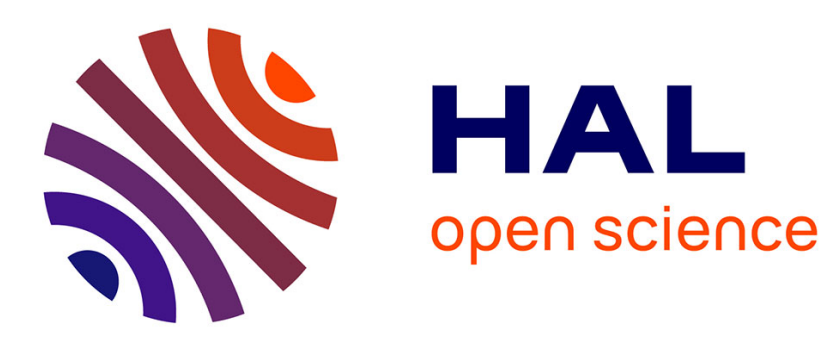

\title{
L'insertion professionnelle dans le milieu de l'ingénierie: une question de genre
}

Biljana Stevanovic

\section{To cite this version:}

Biljana Stevanovic. L'insertion professionnelle dans le milieu de l'ingénierie: une question de genre. Biennale internationale de l'éducation, de la formation et des pratiques professionnelles, Jul 2012, Paris, France. halshs-00866594

\section{HAL Id: halshs-00866594 https://shs.hal.science/halshs-00866594}

Submitted on 26 Sep 2013

HAL is a multi-disciplinary open access archive for the deposit and dissemination of scientific research documents, whether they are published or not. The documents may come from teaching and research institutions in France or abroad, or from public or private research centers.
L'archive ouverte pluridisciplinaire HAL, est destinée au dépôt et à la diffusion de documents scientifiques de niveau recherche, publiés ou non, émanant des établissements d'enseignement et de recherche français ou étrangers, des laboratoires publics ou privés. 


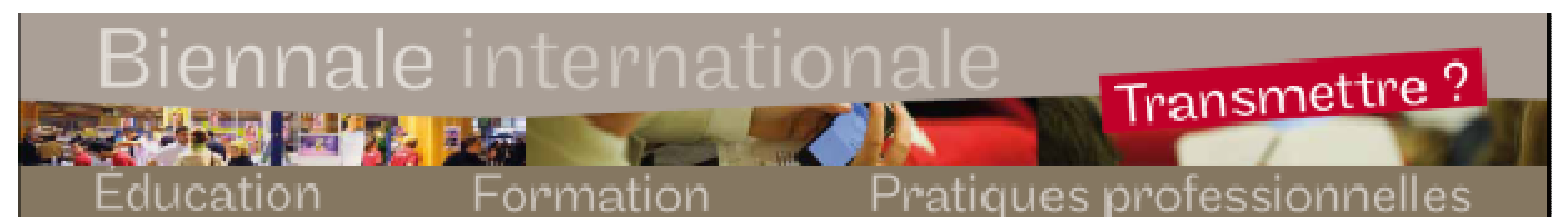

Communication $n^{\circ} 49$ - Atelier 32 : Métiers de l’industrie

\title{
L'insertion professionnelle dans le milieu de l'ingénierie : Une question de genre
}

\author{
Biljana Stevanovic, chercheur, CERSE, Université de Caen .
}

Résumé: En s'appuyant sur les enquêtes «Premier Emploi » réalisées en 2008 six mois après l'obtention de diplôme auprès de la promotion 2007 et les entretiens semi-directifs, cette communication a pour objectif d'étudier la construction de l'identité professionnelle des femmes et des hommes diplômé-e-s de l'EPF-Ecole d'ingénieurs dans la période de l'insertion professionnelle. L'analyse des résultats permet de conclure que les femmes ingénieurs diplômées de l'EPF ont une insertion professionnelle globalement plus favorable que les hommes diplômés de la même école en termes de rapidité d'insertion, de chômage, d'accès au statut de cadre et de part en CDI. Cependant, les femmes diplômées de cette école n'échappent pas à la ségrégation liée à l'accès aux postes de responsabilités, ou aux stéréotypes liés à l'articulation travail-famille.

Mots clés: insertion professionnelle - identité professionnelle - ingénieurs - genre.

\section{Introduction}

L'insertion professionnelle est un processus qui évoque un passage d'un état à un autre (Dubar, 1991) de l'école à l'emploi, et celui d'un statut d'âge, de l'adolescence à un autre, l'âge adulte (Galland, 1991). L'insertion professionnelle est aussi un phénomène qui sousentend une transition: les processus d'accès des jeunes à un emploi dépendent avant tout de la structuration des marchés du travail par les politiques de main-d'œuvre des entreprises et par l'intervention de l'État (Rose, 1998). Pour Agulhon (2006) l'insertion professionnelle est un mode d'évaluation du système de formation qui valide ou invalide les politiques scolaires de la démocratisation et la gestion de la transition professionnelle des jeunes. Cependant, nous ne pouvons pas dissocier l'analyse de l'insertion de celle des relations entre formation et emploi car les transformations des systèmes de formations, le fonctionnement des systèmes d'emploi et du marché du travail permettent comprendre les modes d'insertion (Verdier, 1996).

Aujourd'hui l'insertion n'est pas le simple passage de l'école à l'emploi, elle est un cheminement complexe de situations diverses (chômage, stages, emplois précaires, emplois déclassés) que traversent les jeunes diplômés. Cette période, plus ou moins longue et conditionnée par des facteurs tels que, l'âge, le sexe, le niveau de diplôme, la spécialité de diplôme, l'origine sociale et les réseaux sociaux, construit des parcours professionnels et sociaux (Agulhon, 2006). La socialisation des jeunes dans le cadre de l'insertion témoigne de leur identité professionnelle construite à partir des expériences vécues dans les interactions avec les institutions et les acteurs qu'ils rencontrent (Dubar, 2001). 
Les discriminations liées à l'origine sociale, à l'origine ethnique et au sexe, perdurent tout au long de la vie active (Agulhon, 2006). Plusieurs études (Marry, 2004; Laufer, 2005; Belle, 1991) ont montré que le monde de l'ingénierie est un monde dominé par des stéréotypes sexués, ce qui rend plus difficile l'insertion des femmes. Les éléments propres aux sciences et génie, la culture et les normes du milieu, ainsi que les stéréotypes et perceptions auraient un impact négatif sur la présence des femmes dans ce domaine. (Myrand, 2008) La masculinité présente dans le milieu renforcent les stéréotypes et la perception des femmes que le milieu de l'ingénierie est développé par et pour les hommes, ce qui pourrait expliquer les difficultés des femmes à s'y intégrer. Les hommes composant la majorité des effectifs ont formé le domaine à leur image, valorisant l'indépendance, la compétitivité, la performance, la prise de risque, l'agressivité et la rationalité, toutes des caractéristiques traditionnellement associées aux hommes. Même si nous notons une évolution et une ouverture, la profession d'ingénieur semble préserver les formes culturelles des pratiques institutionnelles qui intimident les femmes.

Dans le secteur des grandes écoles d'ingénieurs, même si elles restent minoritaires, leur pourcentage passe de 18,2 en 1984, à 19,6 en 1989, à 22,6 en 1994, à 22,3 en 1998, à 23,1 en 2000 , puis à $25 \%$ en 2008. Mais, la ségrégation éducative prend la forme de la ségrégation professionnelle au moment de l'entrée sur le marché du travail. Même si le nombre de femmes ingénieurs diplômées augmente en France, leur proportion reste faible et représente 17,7\% des 662800 ingénieurs diplômés de moins de 65 ans. Aussi le nombre de femmes diplômées ingénieurs en activité dans les grandes entreprises (publiques et privées) ayant le statut de cadre s'élève à 16,7\% (342200). (Women's Forum, 2008, p. 9) Les femmes ingénieurs diplômées sont le plus représentées en «agronomie, sciences de la vie et agro-alimentaire » $(42 \%)$ et en chimie (32\%). Par contre, nous comptons $21 \%$ de femmes en «physique, matériaux fluides», $12 \%$ en «sciences et technologie de l'information et de la communication », $12 \%$ en «génie civil, BTP, mines, géologie » et $9 \%$ en «mécanique, production, productique ». (Women's Forum, 2008, p.11)

Cette communication a pour objectif d'étudier l'insertion professionnelle des femmes et des hommes diplômé-e-s de l'ex-Ecole Polytechnique Féminine aujourd'hui «EPF-Ecole d'ingénieurs ». Il s'agit de comprendre le contexte de l'insertion professionnelle et éventuelles difficultés rencontrées des diplômé-e-s d'une école d'ingénieurs de deuxième rang. Les femmes et les hommes ont-ils la même insertion après leur sortie de l'école ? Ont-ils rencontré les mêmes difficultés? Leurs carrières progressent-elles de la même façon? Quelle identité professionnelle les femmes construisent-elles dans cette phase de transition?

Dans un premier temps, nous nous attacherons à présenter la revue de la littérature sur la question de l'insertion professionnelle et de l'évolution des carrières des femmes en ingénierie. Dans un deuxième temps, nous présenterons les résultats de l'enquête auprès des diplômés de l'EPF-Ecole d'ingénieurs, en mettant en évidence les différences hommes/femmes, pour les rapprocher, par la suite, de ceux obtenus au niveau national.

\section{Insertion professionnelle et carrières des femmes en ingénierie: un bilan contrasté}

Si différentes recherches ont montré, dans les années 70, les difficultés de l'insertion des femmes diplômées ingénieurs sur le marché de l'emploi (Peslouan, 1974) et des perspectives de carrière moins favorables aux femmes dans les métiers techniques de l'industrie, il semble qu'aujourd'hui les femmes ingénieurs subissent moins de discrimination au moment de 
l'embauche. Epiphane et Martinelli (1997) ont montré que l'insertion professionnelle des jeunes femmes diplômées du supérieur et particulièrement des écoles de commerce et d'ingénieurs est l'une des plus rapides. La durée de recherche d'emploi des diplômés des écoles d'ingénieurs est l'une des plus courtes : $98 \%$ d'entre eux accèdent à un emploi en moins de six mois. Cependant, cette enquête montre que les jeunes femmes ingénieurs sont légèrement moins nombreuses que les hommes à accéder à leur premier emploi dans ces délais. Une partie des diplômés des écoles d'ingénieurs est confrontée au chômage, avant d'accéder à leur premier emploi, 50\% des jeunes femmes ingénieurs connaissent le chômage contre 32\% des hommes. (Epiphane et Martinelli, 1997, p.117)

Aujourd'hui, les femmes sont toujours proportionnellement plus nombreuses que les hommes à se retrouver à la recherche d'emploi à la sortie de l'école. D'après les résultats de la 18e enquête sur l'insertion professionnelle des diplômés des grandes écoles auprès de la promotion 2007 , six mois après la sortie de l'école, $13,6 \%$ des femmes ingénieurs sont à la recherche d'emploi contre 6,5\% des hommes ingénieurs. (CGE, 2008, p.10) Nous remarquons que les jeunes femmes ont une part de temps passé au chômage légèrement supérieure à celle des hommes mais il faut cependant noter que la majorité de femmes occupent un emploi : 83\% des femmes ingénieurs contre $92 \%$ des hommes. (CNISF, 2007, p.103)

Les femmes sortant des grandes écoles sont aussi plus nombreuses à occuper les postes à durée déterminée (CDD). D'après l'étude réalisée par Epiphane et Martinelli (1997) le recours au CDI (contrat de durée indéterminée) des sortants des écoles d'ingénieurs est plus fréquent pour les hommes que pour les femmes (80\% contre 74\%). (Epiphane et Martinelli, 1997, p.117) En 2008, le taux de recours au CDI s'élève à $72 \%$ pour les femmes et à $84 \%$ pour les hommes ingénieurs.(CGE, 2008) En ce qui concerne les femmes ingénieurs, aujourd'hui, elles subissent moins de discriminations au moment d'embauche, et elles s'insèrent plus rapidement sur le marché de l'emploi que les femmes diplômées des filières tertiaires et littéraires. Il faut souligner aussi, qu'aujourd'hui, en France, un diplôme d'ingénieur est un atout sur le marché du travail. Les opportunités d'emploi dans ces métiers sont nombreuses et significatives pour les hommes mais pour les femmes aussi. Plus de neuf femmes ingénieurs sur dix sont en activité $(91,9 \%)$ et $85,2 \%$ en activité professionnelle ont un statut de cadre. (Women's Forum, 2008) Parmi les femmes ingénieurs moins de 65 ans, 5,5\% sont à la recherche d'un emploi alors que la moyenne nationale est de 8,9\% (ensemble des femmes de moins de 65 ans).

Dans une enquête menée auprès des polytechniciens et des polytechniciennes des promotions 1972-1990, Marry (1995) fait état de différences dans les carrières des uns et des autres. La première constatation qui ressort de cette étude est que la possession d'un diplôme élevé leur permet d'accéder aux positions de cadre supérieur, ainsi que de les protéger du chômage. Aucune polytechnicienne ne déclare être au chômage, mais par contre cette situation concerne trois polytechniciens.

Mais nous devons souligner aussi que, même s'il y a des progrès, des différences persistent entre les jeunes hommes et les jeunes femmes au début de la vie active. Dans une enquête menée auprès des diplômés en 1998 de 3e cycles universitaires et d'écoles d'ingénieurs en physique, génie industriel, automatisme, informatique industrielle, mécanique, électricité, électronique, informatique et traitement des réseaux, en emploi en 2001, trois ans après la sortie de l'école, Epiphane (2006) a montré que les jeunes femmes scientifiques au cours des trois premières années de la vie active ne rentabilisent pas de la même manière leur formation selon le segment professionnel qu'elles parviennent à investir. En effet, les femmes occupent moins souvent que les jeunes hommes scientifiques les fonctions pour lesquelles ont été 
formées et les disparités sont importantes pour les scientifiques qui n'ont pas atteint «les emplois cœur ${ }^{1}$ des segments professionnels. Seulement la moitié des jeunes femmes scientifiques contre deux tiers des hommes occupent, trois ans après leur entrée sur le marché du travail, un emploi directement lié à leur formation initiale ( $51 \%$ contre $66 \%$ ). Elles sont, par contre, plus nombreuses à occuper les «emplois périphériques » $(23 \%$ contre $18 \%)$ et beaucoup plus fréquemment d' «autres emplois » $(26 \% \text { contre } 16 \%)^{2}$. Parmi les femmes qui occupent les «autres emplois » seulement $48 \%$ n'ont jamais connu le chômage contre $72 \%$ des hommes et $66 \%$ des femmes qui occupent les «emplois cœur». Mais quand les femmes arrivent à atteindre les «emplois cœur», elles obtiennent des conditions d'emplois et des salaires tout à fait comparables à ceux des jeunes hommes scientifiques.

Nous avons vu plus haut qu'il y avait de plus en plus de filles en école d'ingénieur et certaines, qui sont entrées dans le monde professionnel, occupent parfois de hauts postes de cadre. Mais beaucoup de recherche ont montré que les femmes progressent moins vite que les hommes dans leurs carrières. Dans l'enquête auprès des polytechniciens, Marry (1995) précise que les polytechniciennes occupent moins souvent que les polytechniciens les postes de direction, d'encadrement, les «positions hiérarchiques les mieux rémunérées ». En effet, les deux tiers des polytechniciennes ont des personnes sous leur responsabilité directe, un quart dirige des équipes composées de 10 personnes ou plus. Mais, toutefois, il faut rappeler qu'en 1994, 38\% des polytechniciennes ont réussi à accéder à ces postes de direction contre 53\% des polytechniciens. Ces résultats confirment les conclusions de Bertin-Maurot (1997) sur la participation des femmes à l'exercice du pouvoir dans les grandes entreprises françaises, qui constate que les femmes ne sont pas représentées aux hauts postes de la sphère économique, jamais plus de 7\%. En 2007 le taux de féminisation dans les équipes de direction des entreprises privées de plus de 200 salariés est d'environ $8 \%$ et de $11 \%$ dans les grandes entreprises publiques. (Vincent, 2010)

L'association "Grandes écoles au féminin" (GEF), qui regroupe des femmes diplômées des neuf plus grandes écoles en France (l'ENA, HEC, Centrale ou Polytechnique... ), a effectué une enquête en 2006 auprès de 7200 diplômés ( $65 \%$ d'hommes et $35 \%$ de femmes). Cette enquête montre que les femmes parviennent moins fréquemment à des postes de responsabilités : seules $15 \%$ des femmes trentenaires occupent une position de dirigeant (19 $\%$ chez les hommes). Passé 40 ans, elles sont $27 \%$, contre $35 \%$ chez les hommes. A diplôme et âge égaux (41-50 ans), $28 \%$ d'entre elles siègent au comité de direction, (43\% chez les hommes). Quand les femmes parviennent à occuper les postes d'encadrement, une proportion plus importante encadre des petites équipes : $18 \%$ des femmes ont sous leur autorité un groupe de plus de 50 personnes, contre $32 \%$ des hommes. Ces résultats confirment les conclusions de l'enquête de CNISF (2007). Selon cette enquête, 20\% des femmes moins de 30 ans ont des responsabilités hiérarchiques. Parmi celles qui exercent des fonctions d'encadrement, $15 \%$ (contre $20 \%$ des hommes) encadrent une petite équipe, $4 \%$ encadrent un service ou un département (contre $7 \%$ des hommes) et $0 \%$ a des fonctions de direction générale (contre 1\% des hommes). (CNISF, 2007, p.37)

Comment expliquer les difficultés des femmes ingénieurs à progresser dans leur carrière? Plusieurs auteurs soulignent que le fonctionnement organisationnel de l'entreprise écarte les

\footnotetext{
1 Les « emplois coeur» sont des emplois auxquels une grande partie des diplômés d'une filière se destinent après leur formation et dont le contenu est proche de celui de l'emploi atteint

2 Les «emplois périphériques» rassemblent les emplois occupés par quelques diplômés dont le contenu d'activité reste proche de la formation initiale. Les «autres emplois » regroupent ceux dont le contenu d'activité est éloigné du contenu de la formation initiale.
} 
femmes des postes de direction (Laufer, 1982, 2005; Belle, 1991; Evetts, 1998; Roberts et Ayre, 2002).

Une étude particulièrement intéressante, auprès de soixante femmes cadres et trente cadres masculins, répartis dans treize grandes entreprises, menée par Laufer, en 1982, a souligné les différentes modalités du fonctionnement traditionnel de l'entreprise qui neutralise la féminité à travers une exigence de participation au modèle masculin. D'après Laufer (1982), les femmes entre l'égalité et la différence « reconnaissent et acceptent «leur différence », celle d'avoir à être non seulement efficaces mais «féminines », celle d'avoir en charge une famille et d'avoir un rôle qui ne les autorise pas à investir leur carrière comme le ferait un homme » (p.67). Citons à ce propos les analyses de Belle (1991) qui sur l'exemple de trois grandes entreprises de Grenoble, spécialisées en électricité et électronique, a mis en évidence l'influence que la culture de l'entreprise exerce sur les carrières des femmes. L'auteure conclut que la possibilité de la progression des carrières et l'accès aux postes de pouvoir dépend des hommes cadres et du modèle que l'entreprise propose, «la négociation entre l'entreprise et la femme cadre respecte deux règles implicites : l'équivalence sociale par la compétence et la différence par le statut sexuel » (p.183).

D'après une étude auprès des quinze femmes ingénieurs en Angleterre, Evetts (1998) a montré que les femmes s'adaptent facilement à la nature du travail exigé dans le domaine de l'ingénierie, mais qu'elles ont beaucoup plus de difficultés à s'intégrer à la culture organisationnelle dans les entreprises. L'auteure souligne que l'adaptation à la culture masculine est l'aspect du travail le plus exigent pour les femmes ingénieurs. Elle suggère aussi que les valeurs et croyances présentes relevant de la culture typiquement masculine ainsi que les stéréotypes créent des conditions de travail particulièrement hostiles aux femmes. Les dirigeants dans les organisations continuent à avoir des perceptions très sexuées à l'égard des femmes et préfèrent qu'elles entreprennent d'autres voies que l'encadrement des équipes.

"The women enjoyed the practice of engineering and found it easy to reconcile being a woman and doing engineering work. The problems that some of the women experienced arose because of aspects of gender in the work organisation rather than because of technical skills required to be an engineer. » (Evetts, 1998, p.289)

Dès lors, nous pouvons nous demander qui sont les femmes qui parviennent à atteindre les hautes sphères de la hiérarchie des entreprises. Laufer (2005), dans une recherche auprès de quatre entreprises françaises, a étudié les facteurs qui permettent aux femmes cadres de franchir le «plafond de verre ». Selon l'auteure le plafond de verre apparaît possible comme le résultat de l'interaction complexe entre processus organisationnel et stratégie des femmes. Les cadres à potentiel «sont des cadres ayant atteint un certain niveau de responsabilité ou susceptibles de l'atteindre » (p.35) avant l'âge de 35 ans et pour qui il est possible de développer un parcours professionnel qui les conduira vers les postes de directeur d'un service, d'une unité, d'une filiale. Les femmes interrogées déclarent que pour réussir il s'agit d'être déterminée, de prendre leur carrière en main et surtout de provoquer les opportunités. La compétence à manager les équipes ainsi que la mobilité et la disponibilité sont soulignées comme favorables à l'évolution de carrière. La possibilité de la réussite est décrite comme l'union des éléments suivants : une très bonne santé, un très bon équilibre des enfants, un soutien sans faille du compagnon, une bonne organisation domestique. Les facteurs qui peuvent limiter leur capacité à poursuivre leur franchissement du plafond de verre concernent en grande partie leur vie familiale, leur mobilité, leur disponibilité rendues difficiles si les négociations conjugales jouent en leur défaveur. Pour Evetts (1998) aussi, les difficultés des 
femmes ingénieurs d'accéder aux postes des responsabilités dépendent de la sphère familiale, alors que la disponibilité, les capacités à travailler dur et l'adaptation à la culture organisationnelle existante de l'entreprise pourraient être associées positivement à l'évolution de leurs carrières.

Un autre indicateur d'inégalités entre les hommes et les femmes dans l'ingénierie est le salaire. Les salaires des hommes sont supérieurs à ceux des femmes. Les résultats montrent des différences de salaires très nettes qui s'accroissent avec l'âge. Les écarts de salaires, qui commencent dès les premières années de travail, se renforcent au cours de la carrière : l'écart des salaires des débutants est de $7,5 \%$ et s'accroît pour atteindre $24 \%$ entre 45 et 49 ans. (Women's Forum, 2008, p.30) Cet écart de $24 \%$ pourrait s'expliquer par le fait que les femmes occupent à cette tranche d'âge moins souvent des fonctions de direction. Des écarts sont deux fois plus importants dans le privé que dans le public. En 2007, nous notons dans le public un écart de $23 \%$, alors que dans le privé, par exemple, cet écart s'élève à $47 \%$ dans l'industrie chimique, à $41 \%$ dans l'industrie agro-alimentaire et à 45\% dans l'industrie textiles, habillement, chaussures. (Women's Forum, 2008, p.34)

Le métier d'ingénieur est considéré comme un métier qui rend difficile l'articulation de la vie professionnelle et de la vie familiale. De nombreuses études ont souligné les difficultés des femmes à articuler la vie professionnelle et la vie familiale (Peslouan, 1974; Marry, 2004; Duru-Bellat, 2004; Stevanovic, 2006). D'après une enquête effectuée auprès des élèves de l'ex-Ecole Polytechnique Féminine, Stevanovic (2006) a conclu que les filles sont proportionnellement plus nombreuses à déclarer qu'elles accepteraient de renoncer à la progression de leur carrière à cause des contraintes familiales. Elles sont prêtes à mettre leurs carrières entre parenthèses et à élever leurs enfants en travaillant à temps partiel ou en cessant leur activité professionnelle temporairement, ce qui n'est bien sûr le cas d'aucun garçon. Dans une étude exploratoire canadienne (Van Nieuwenhuyse, Descarries et Deschênes, 2003) auprès de 11 femmes ingénieures âgées de 29 à 44 ans, les auteurs ont étudié le rapport que ces femmes entretiennent avec leur profession ainsi que l'impact de leur milieu de travail sur l'articulation de leur vie professionnelle et de leur vie familiale. Il apparaît que les femmes plus âgées affirment que le fait d'être une femme et mère, peut avoir un impact négatif sur la trajectoire professionnelle en freinant ou en ralentissant la progression de leur carrière. Même si toutes les femmes affirment assumer le double rôle de mère et ingénieur, elles croient que la charge familiale leur revient.

Malgré quelques difficultés, les femmes ingénieurs déclarent, en général, aimer la profession d'ingénieurs, elles sont satisfaites de leur expérience et expriment le plaisir à exercer leur profession (Marry, 2004; Epiphane, 2006; Van Nieuwenhuyse, Descarries et Deschênes, 2003; Evetts, 1998). Cependant quelques-unes interprètent beaucoup plus négativement leur expérience de minoritaires au sein de la profession. Elles ont souligné avoir dû se battre, à leur début, pour s'imposer et se faire respecter en tant que les femmes ingénieurs (Van Nieuwenhuyse, Descarries et Deschênes, 2003).

\section{Problématique et Méthodologie}

L'ex-Ecole polytechnique Féminine, aujourd'hui l'EPF-Ecole d'ingénieurs, est une école en cinq ans, avec prépas intégrées. Cette école d'ingénieurs, fondée en 1925 et destinée aux filles, forme des femmes ingénieurs pendant 69 ans avant de devenir mixte en 1994 en s'ouvrant aux garçons. Aujourd'hui l'école compte 33\% des filles. Depuis l'année 1999 les 
premières promotions mixtes sortent de l'école. La scolarité s'organise en trois cycles : un cycle préparatoire ( 2 premières années de la formation), un cycle d'ingénieurs ( $3{ }^{\text {ème }}$ et $4^{\text {ème }}$ année de la formation) et un cycle d'approfondissement avec obligation de choisir une spécialité parmi les 8 proposées (qui commence en $4^{\text {ème }}$ année et se poursuit en $5^{\text {ème }}$ année). L'école forme des ingénieurs généralistes. La spécialisation choisie détermine le plus souvent le secteur d'activité du premier emploi. L'école en propose huit: aéronautique et espace, énergétique et environnement, ingénierie d'affaires et de projets, management des systèmes d'information, mécanique des matériaux et des structures, ingénierie industrielle et logistique, système et réseaux informatiques, télécommunications. Les élèves entrent à l'école en général avec des mentions «Passable » et «Assez Bien ». Pour la majorité des élèves, leurs parents disposent de titre de l'enseignement supérieur (65\% des pères et $54 \%$ des mères), $35 \%$ des pères exercent la profession d'ingénieur et de cadre d'entreprise alors que c'est le cas de seulement $7 \%$ des mères. (Stevanovic, 2006) L'EPF est une école privée, de « deuxième rang » dont la scolarité est payante. En 2010, selon le classement de «Express » (7 janvier 2010), l'EPF occupe le $11^{\text {ème }}$ rang dans le classement des écoles d'ingénieur après le bac.

Aujourd'hui en France, un diplôme d'ingénieur est un atout sur le marché du travail. Les plus grandes écoles comme l'Ecole polytechnique, l'Ecole des mines, l'Ecole des ponts et chaussées, etc., ouvrent la possibilité de très grandes carrières pour les hommes mais pour les femmes aussi. Si nous avons vu plus haut que le diplôme d'ingénieur permet aux femmes de s'insérer assez rapidement sur le marché du travail et d'accéder au titre de cadre dès leur premier emploi, doit-on supposer que ces nouvelles générations de femmes ingénieurs, issues de cette école de deuxième rang, auront des conditions d'insertion semblables à leurs homologues masculins? Nous faisons l'hypothèse que l'identité professionnelle des femmes diplômées de cette grande école d'ingénieurs de deuxième rang, qui se construit dans ce contexte professionnel, à savoir dans le milieu d'ingénierie, n'échappe pas aux stéréotypes liés à l'articulation travail-famille, aux postes de responsabilité et au salaire.

Nos données sont issues de l'analyse secondaire des enquêtes «Premier Emploi » réalisées par l'EPF-Ecole d'ingénieurs en 2008, six mois après la sortie de l'école auprès de la promotion 2007 et des entretiens semi-directifs. Pour la promotion 2007 nous possédons 157 réponses ( $66 \%$ des hommes et $34 \%$ des femmes). Le taux de réponse aux questionnaires s'élève à $77,7 \%$.

Les entretiens semi-directifs au nombre de vingt (onze femmes et neuf hommes) ont été effectués auprès des jeunes diplômés en situation professionnelle qui ont accepté de nous rencontrer entre 2007 et 2009. D'une durée moyenne d'environ une heure, ils ont donné lieu à une analyse thématique. L'âge minimum est 24 ans et l'âge maximum est 33 ans. Seulement quatre femmes et deux hommes rencontrés ont des enfants, trois femmes sont mariées mais sans enfants, et le reste de l'échantillon est soit célibataire soit en couple. Parmi les quatre femmes qui ont des enfants, l'une d'entre elles travaille à temps partiel dans l'entreprise de son mari, les deux travaillent à temps plein et la quatrième est en congé de maternité mais envisage de travailler à temps plein.

Les indicateurs retenus pour décrire l'insertion professionnelle des ingénieurs EPF sont: le délai de recherche du premier emploi, le moyen de recherche pour trouver l'emploi, le statut d'emploi (cadre), la nature du contrat de travail (CDD, CDI), la fonction exercée, le salaire, l'intégration dans l'entreprise, la satisfaction du métier exercé et l'articulation travail-famille. La question de l'insertion professionnelle exige la prise en compte des conditions d'accès à l'emploi des différentes catégories sociales par sexe. Signalons que 
l'enquête sur laquelle nous nous appuyons ne prend pas en compte la variable «origine sociale ». Ceci constitue une limite à notre travail compte tenu de l'importance de l'appartenance sociale aux processus de la réussite scolaire, de l'orientation scolaire et de l'insertion professionnelle. Cependant, comme nous l'avons souligné plus haut, lors de l'enquête auprès des élèves de l'école, Stevanovic (2006) a constaté que pour la majorité des élèves leurs parents sont issus des milieux de cadres supérieurs.

\section{L'insertion professionnelle des diplômé-e-s de l'EPF-Ecole d'ingénieurs}

\section{Situation professionnelle à la sortie de l'école}

Aujourd'hui en France, un diplôme d'ingénieur est un atout sur le marché du travail, pour les hommes mais pour les femmes aussi, puisque $91 \%$ des femmes sont en activité professionnelle. (CGE, 2008) Ces résultats se confirment sur l'exemple de l'EPF-Ecole d'ingénieurs. Même s'il s'agit d'une école de deuxième rang, leur diplôme d'ingénieur leur permet de s'insérer assez rapidement sur le marché du travail et d'accéder au titre de cadre dès leur premier emploi. Les opportunités d'emploi des diplômés de l'EPF-Ecole d'ingénieurs sont significatives pour les deux sexes. Cependant il faut noter que six mois après la sortie de l'école leur insertion sur le marché du travail semble être moins favorable que la moyenne nationale. 65\% de la promotion 2007 de l'EPF est en activité professionnelle contre $72 \%$ au niveau national. Comme nous notons une proportion importante des diplômés en poursuite d'études, nous pensons que les diplômés de l'EPF éprouvent le besoin de compléter leur formation (21\%).

Tableau 1. Situation professionnelle à la sortie de l'école des diplômé-e-s de la promotion 2007

\begin{tabular}{|l|l|l|l|}
\hline$\%$ & Hommes & Femmes & Total \\
\hline En activité professionnelle & 65,0 & 66,6 & 65,6 \\
\hline En recherche d'emploi & 7,7 & 11,1 & 8,9 \\
\hline En poursuite d'étude & 22,3 & 18,5 & 21,0 \\
\hline Sans activité & 2,9 & 3,7 & 3,1 \\
\hline Non réponses & 1,9 & 0 & 1,2 \\
\hline Total & 100 & 100 & 100 \\
\hline
\end{tabular}

Nous avons supposé que ces nouvelles générations de femmes ingénieurs, issues de cette école de deuxième rang, ont les mêmes conditions d'insertion et d'évolution de carrière que leurs collègues hommes. Il apparaît que les femmes diplômées de cette école s'insèrent plus rapidement sur le marché du travail que les hommes diplômés de la même école puisque la proportion de femmes en activité professionnelle est plus élevée : 66,6\% des femmes contre $65 \%$ des hommes. Par contre, au niveau national, les hommes ingénieurs semblent être plus avantagés puisque $74,3 \%$ déclarent être en activité professionnelle contre 67,5\% des femmes (CNISF, 2007).

En ce qui concerne la recherche d'emploi, nous remarquons qu'un nombre important est en recherche d'emploi $(8,9 \%)$, mais ce pourcentage se rapproche de la moyenne nationale. Il semble que les jeunes femmes soient plus affectées par le chômage, $11,1 \%$ contre 7,7\%. Même si au niveau national, nous retrouvons les mêmes proportions, il apparât que les femmes diplômées de l'EPF sont moins touchées par le chômage que les femmes ingénieurs issues des autres écoles. 


\section{Premier emploi}

Les résultats montrent que les écarts hommes/femmes sont limités sur les indicateurs du «Premier emploi ». Cependant, il apparaît que les femmes sont plus nombreuses à accéder à un premier emploi par le biais de stage et qu'elles sont aussi plus nombreuses à être recrutées en CDI.

L'étude de délais de recherche du premier emploi des diplômés de l'EPF-Ecole d'ingénieur montre que la majorité de ceux qui travaillent a signé un contrat avant la fin de leur formation, à l'issu de leur stage dans le cadre de leur formation d'ingénieur (59,5\%), et que les femmes sont plus nombreuses à accéder à l'emploi par le biais de stage $(61,7 \%$ contre $58,3 \%$ ). $25,5 \% \%$ ont signé leur premier contrat dans les deux mois qui ont suivi la sortie de l'école, $13,8 \%$ de deux à quatre mois après la sortie de l'école et $1 \%$ de quatre à six mois après la sortie de l'école. Parmi les diplômés qui ont signé leur contrat dans les deux mois, de deux à quatre, et de quatre à six mois qui ont suivi leur sortie de l'école, nous ne notons pas de différences particulières entre hommes et femmes.

Le secteur privé est le principal employeur des diplômés de l'EPF. La majorité des diplômés est salariée dans une entreprise privée (93,2\%) et très peu dans le secteur public $(1,9 \%)$. Nous retrouvons un peu près le même nombre d'hommes et de femmes salariés dans le secteur privé alors que nous notons 2,9\% des hommes dans le secteur public et aucune femme. Il y a très peu de diplômés qui se sont lancés dans la création de l'entreprise, un peu près d'un pourcent, et il s'agit surtout des hommes.

L'étude de nature de contrat nous indique que la majorité de diplômés est en CDI (Contrat de durée indéterminée): 79,6\%. Un pourcentage moins significatif est en CDD (Contrat de durée déterminée): $11,6 \%$. En ce qui concerne la situation par sexe, on remarque l'écart de taux de recours au CDI. Le recours au CDI est plus fréquent pour les jeunes femmes alors que l'écart est limité sur le recrutement en CDD. Ainsi nous notons que 86,1\% des femmes contre $76,1 \%$ des hommes sont en CDI et $11,9 \%$ des hommes contre $11,1 \%$ des femmes en CDD. Il convient de souligner que le taux de recours au CDI est plus fréquent pour les jeunes femmes diplômées de l'EPF que pour les femmes ingénieurs au niveau national $(72 \%)$.

Dès le premier emploi les femmes ingénieurs EPF accèdent au plus grand nombre au statut de cadre. Ces résultats sont contradictoires car au niveau national pour la promotion 2007 nous comptons $95 \%$ d'hommes contre $88 \%$ de femmes cadres (CGE, 2008) alors que 94,4\% de femmes ingénieurs EPF déclarent avoir le statut de cadre contre 76,1\% d'hommes. Rappelons aussi que Epiphane (2006) a constaté que trois ans après la sortie de l'école, $51 \%$ des femmes scientifiques contre $66 \%$ d'hommes occupent un emploi connecté à leur filière d'origine, appelés « emplois cœur».

\section{Salaire}

Les résultats de toutes les études (Marry, 1995, 2004; Bertin-Mourot, 1997; Laufer, 1984, 2005; Belle, 1995; Evetts, 1998; Roberts et Ayre, 2002; CNISF, 2007; CGE, 2008) s'accordent sur le fait que les femmes ingénieurs soient pénalisées au niveau salarial. Ces résultats se confirment partiellement sur le cas de l'EPF. Les moyennes de salaire brut annuel sont égalitaires entre les hommes et les femmes puisque le salaire brut moyen des hommes s'élève à $32506 €$ et celui des femmes à $32286 €$. En effet, on n'observe pas de 
différence entre les salaires des hommes et des femmes alors que selon l'enquête de CNISF (2007) l'écart de salaires des débutants est de 7,5\%. Doit-on penser que les femmes diplômées de l'EPF négocient mieux leurs contrats que les femmes diplômées des autres écoles? Cependant, lors d'un entretien une jeune femme reconnaît de ne pas avoir su négocier son salaire. Il faut reconnaître qu'il s'agit du début de leur vie professionnelle et que les écarts se creusent plus significativement après trente ans. (CNISF, 2007; Le Pellec, Roux, 2001) Nous supposons que cette égalité salariale ne persistera pas lors du déroulement de leurs carrières, parce que la différence de salaire après trente ans est liée à un moindre accès des femmes aux postes d'encadrement. Ces écarts de salaire qui s'intensifient avec l'âge pourraient aussi être liés à la sous-estimation ou au manque de confiance des femmes qui n'oseraient pas demander des augmentations ou des promotions.

\section{Fonction occupée, satisfaction et intégration dans l'entreprise}

Dans l'enquête auprès des diplômés de l'EPF, les résultats indiquent que les jeunes ingénieurs de deux sexes occupent les mêmes fonctions, ainsi que les progressions hiérarchiques identiques. Ils exercent principalement dans le bureau d'études (34\%), dans la production $(17,4 \%)$ et en tant que «commercial, technico-commercial, ingénieur d'affaires » $(17,4 \%)$. Même si nous ne notons pas de différences significatives entre les fonctions des hommes et des femmes, il apparaît que les femmes exercent plus que les hommes dans la recherche $(16,6 \%$ des femmes contre $10,4 \%$ des hommes). Lors des entretiens, les femmes et les hommes diplômé-e-s EPF déclarent exercer en tant que chef de projet ou encadrer les petites équipes. Au niveau national en début de vie professionnelle, ces résultats se confirment puisque les écarts hommes/femmes sont limités sur plusieurs indicateurs comme "être chef de projet», "prendre des décisions stratégiques » ou « encadrer une petite équipe ». Par contre en fin de carrière les écarts se creusent significativement. Si les écarts sont réduis pour les postes qui n'impliquent pas de grandes responsabilités (animer une équipe, être chef de projet, être un expert), les écarts sont conséquents dès qu'il s'agit d'exercer des fonctions de direction générale, d'avoir des responsabilités de budget ou prendre des décisions stratégiques. (CNISF, 18e enquête, 2007)

D'après les déclarations des hommes et des femmes interrogés, avoir des enfants peut ralentir la carrière de la femme. Deux tendances se dégagent. Les femmes qui ont des enfants soulignent que "les employeurs préfèrent donner des responsabilités aux femmes plus jeunes, parce qu'ils sont sûrs qu'elles n'auront pas d'enfants qu'à celles de trente ans qui ont des enfants». Les femmes plus jeunes, dans la vingtaine, soulignent que les employeurs ne leur donnent pas de responsabilité parce qu'elles sont «soupçonnées » d'avoir des enfants et que de toute façon "il n'y aura pas de carrière avant trente-cinq ans ». Une jeune femme de 27 ans qui venait de se marier a failli se voir attribuer sa mission d'aller en Afrique à quelqu'un d'autre parce que son employeur croyait qu'elle était enceinte : «je me suis juste mariée. Je ne vais pas avoir d'enfants. » Laufer (2005) a constaté que les cadres à potentiel, susceptibles d'accéder aux postes d'encadrement et hautes fonctions, sont des cadres ayant atteint un certain niveau de responsabilité avant l'âge de trente-cinq ans. Si les femmes doivent attendre l'âge de trente-cinq ans pour faire carrière et si commencer à faire carrière après trente-cinq ans est déjà tard pour accéder aux postes de responsabilités, doit-on conclure que les femmes ne pourront jamais attraper leur retard? Selon Roberts et Ayre (2002) le réseau masculin détermine informellement les critères de promotion et soutient les candidats à leur image. Les femmes sont en général exclues de ces réseaux et manquent de mentor. D'ailleurs, si elles ont des enfants, 
elles sont perçues comme moins engagées dans leurs carrières que les hommes. Ces résultats se confirment sur l'exemple de notre étude. D'abord, même si les femmes déclarent que leur intégration dans l'entreprise s'est bien passée, elles ont eu besoin d'un mentor masculin pour s'y intégrer. L'exemple de la jeune femme à qui on a promis un poste et que, par la suite, on l'a attribué à un autre homme sans l'avertir, témoigne de son exclusion du réseau masculin. Le cas de la jeune femme qui s'est mariée et qui était soupçonnée d'attendre un enfant, montre qu'elle a été tout de suite considérée comme moins engagée, puisque son employeur a voulu envoyer quelqu'un d'autre en mission. En accord avec Roberts, Ayre (2002), cette perception de la femme qui n'est pas assez disponible à cause des enfants, est utilisée pour discriminer toutes les femmes qui exercent le métier d'ingénieur.

Malgré quelques inconvénients et en accord avec d'autres études (Marry, 2004; Epiphane, 2006, Van Nieuwenhuyse, Descarries et Deschênes, 2003; Evetts, 1998), les femmes diplômées de l'EPF que nous avons rencontrées déclarent être satisfaites d'exercer le métier qu'elles aiment. Elles évoquent le plaisir du métier et la sécurité de l'emploi alors que les hommes évoquent les opportunités de développement de leurs carrières, les possibilités d'exercer les responsabilités et de voyager. Contrairement à l'étude australienne qui indique que les femmes dans la trentaine envisagent de quitter la profession (Roberts et Ayre, 2002), aucune femme rencontrée n'évoque cette possibilité. Dans les entretiens, les hommes et les femmes interrogés déclarent, pour la plupart, être bien intégrés dans leur entreprise. Les jeunes femmes déclarent ne pas avoir subi de discrimination de la part de l'équipe et parlent de leur intégration comme quelque chose qui s'est passée de façon naturelle: «Ils m'ont tous pris comme leur petite sæur gentiment, ils m'ont tous aidé, pour eux je suis une collègue de travail ». Ces résultats sont contradictoires aux études anglo-saxonnes (Roberts, Ayre, 2002; Phipps, 2002) qui démontrent que les femmes ne se sentent pas intégrées dans les équipes masculines de leurs entreprises ni dans les réseaux sociaux masculins. Mais quand on leur pose la question si quelqu'un les a aidées dans l'intégration, elles désignent toujours un homme, un chef d'équipe, un responsable ou un tuteur : "Mon employeur m'a pris sur son ail». Nous supposons que les femmes auraient besoin d'un mentor masculin pour être acceptées par «boy's club». (Roberts, Ayre, 2002)

En revanche, la discrimination à l'égard des femmes se fait ressentir par le refus d'un poste de responsabilité, "ça fait deux fois qu'on me refuse des responsabilités sous prétexte que je suis trop jeune », ou la distribution d'un poste à un homme plus âgé sans avertissement, «le poste m'était promis et on a pris quelqu'un d'autre sans m'avertir», ou encore par un moindre salaire, "mon premier salaire aurait pu être plus haut, c'est peut-être moi qui n'ai pas su négocier ». Ces résultats confirment ceux de Roberts, Ayre (2002) et Phipps (2002), que les femmes ingénieurs ont moins d'opportunités de promotion et de reconnaissance de leurs compétences.

\section{Conclusion}

Au niveau national, pour l'ensemble des indicateurs d'insertion, les résultats montrent que les jeunes femmes sont en retrait par rapport à leurs homologues masculins. Aucun indicateur (activité professionnelle, temps de recherche d'emploi moins de 2 mois, part en CDI, part en cadre, salaire) ne fait exception. Toutefois, il faut reconnaître que les écarts sont limités et que les jeunes femmes semblent avoir des débuts dans le métier semblables 
aux hommes. Dans le cas de l'EPF, au début de la vie active, il apparaît que les femmes diplômées de l'EPF ont une insertion professionnelle globalement plus favorable que les hommes diplômés de la même école en termes de rapidité d'insertion, de chômage, d'accès au statut de cadre et de part en CDI.

Nous pouvons nous demander pourquoi les jeunes femmes diplômées de cette école d'ingénieurs parviennent à avoir une meilleure insertion professionnelle que les jeunes hommes et pourquoi leur insertion sur le marché du travail est meilleure sur certains indicateurs que la moyenne nationale. Nous formulons l'hypothèse que l'origine féminine de l'école aurait un impact sur leur insertion professionnelle, et les aiderait à mieux planifier et à mieux gérer leur carrière. Ainsi leur diplôme d'ingénieur obtenu dans ce cadre particulier serait le vecteur d'égalité homme/femme dans le monde professionnel. D'après une étude réalisée auprès des élèves des premières promotions mixtes de l'EPF, Stevanovic (2006) a conclu que, avec le passage à la mixité, l'école a réussi à garder un cadre où les filles sont bien intégrées, où elles se sentent à l'égalité, à l'aise, à leur place et détachées des stéréotypes qui donnent de l'ingénierie une image masculine. Il est bien sûr difficile de vérifier cette hypothèse d'autant plus que, dans les entretiens, les femmes déclarent ne pas avoir bénéficié d'aide de l'école dans la recherche de leur stage ou de leur premier emploi. D'après les résultats des entretiens, elles déclarent aussi ne pas avoir bénéficié de l'aide du réseau des diplômés EPF en activité professionnelle et que, d'ailleurs, elles étaient rarement contactées par les élèves de l'EPF au sujet d'un stage ou par les jeunes diplômés au sujet d'un emploi. L'hypothèse explicative la plus plausible est que les ingénieures EPF ont plus d'opportunités d'emploi que leurs homologues masculins parce que, avant la mixité, l'école bénéficiait d'une très bonne réputation auprès des entreprises, et que maintenant, quand les employeurs ont le choix entre les femmes venant des autres écoles moins réputées et celles de l'EPF, ils préfèrent recruter les femmes diplômées de l'EPF.

Même si ces constats reposent sur les résultats d'une seule école d'ingénieurs, ils rendent compte de l'insertion professionnelle des diplômés des grandes écoles d'ingénieurs de deuxième rang, en particulier des écoles avec les préparations intégrées. Pour vérifier ces résultats et élargir cette étude, il faudra mener les mêmes enquêtes auprès des hommes et des femmes ingénieurs issus d'écoles semblables en termes de prestige. Pour vérifier si la meilleure insertion professionnelle des femmes est juste la spécificité de cette école aux origines féminines qui compte aujourd'hui le plus grand nombre des filles en France (33\%), ou si d'autres écoles se distinguent par une insertion professionnelle des femmes aussi favorable que l'EPF-Ecole d'ingénieurs, il faudra aussi mener les mêmes enquêtes auprès des écoles qui comptent 30\% des filles (ex. INSA (Instituts Nationaux des Sciences Appliquées): $30 \%$ ).

Dans cet article nous nous sommes intéressée à l'insertion des femmes qui ont réussi à dépasser les déterminismes sociaux et exercent un métier traditionnellement masculin, en l'occurrence le métier d'ingénieur. En assurant aux filles l'accès à l'enseignement secondaire et à l'université, les sociétés occidentales du XXe siècle ont permis leur accès aux filières scientifiques et aux emplois auxquels elles conduisent. Entre 1980 et aujourd'hui, les femmes ont opéré des avancées dans le métier d'ingénieur, mais ces transformations n'ont pas touché tous les domaines de l'ingénierie. Par exemple, nous l'avons vu plus haut, elles sont minoritaires en productique, en informatique, en génie civil, les domaines traditionnellement considérés comme très masculins. Ceci est sans doute lié à des orientations prises dès le lycée. Même si nous notons des progrès 
considérables dans l'orientation des filles vers la filière scientifique au lycée, le choix d'option reste très sexué. Ainsi, dans l'option «SVT », en terminale S, nous comptons $58 \%$ des filles, dans l'option «physique-chimie » la parité est presque atteinte, (47\%), dans l'option «mathématiques » il y a $40 \%$ des filles, alors que dans l'option « sciences de l'ingénieur » les filles sont minoritaires, 14\% (DEPP, 2010).

Cette période de transition de la formation à l'emploi pour ces femmes ingénieurs EPF construit leur identité professionnelle à travers les pratiques quotidiennes, l'organisation institutionnelle des entreprises et les représentations symboliques propres à ce milieu de l'ingénierie. Leur identité professionnelle est fortement influencée par l'image masculine et l'organisation patriarcale des entreprises qui déterminent les critères de promotions. Les femmes diplômées de l'EPF sont satisfaites d'exercer le métier qu'elles aiment. Cependant, elles n'échappent pas à la ségrégation liée à l'accès aux postes de responsabilités ou aux stéréotypes liés à l'articulation travail-famille. Pour mieux comprendre les mécanismes de l'insertion professionnelle dans le milieu de l'ingénierie, il faudra s'intéresser aux expériences accumulées par les étudiantes pendant leur formation et à la construction de leur identité professionnelle pendant leurs études. Les inégalités professionnelles repérées lors de l'insertion des diplômées devront servir pour organiser une meilleure préparation des étudiantes à leur insertion future, en particulier à mieux négocier leurs promotions, leurs salaires et à mieux affronter la culture du milieu de l'ingénierie.

\section{BIBLIOGRAPHIE}

Agulhon Catherine, 2006, «L'insertion professionnelle: un mode d'évaluation du système de formation » in Gérard (dir), Savoirs, insertion, globalisation, (pp.25-38). Paris, Publisud.

Belle Françoise, 1991, Etre femme et cadre. Paris, Harmattan.

Berner Boel, 2003, "L'ingénieur ou le génie du mâle: masculinité et enseignement technique au tournant du XXe siècle », Cahiers du Gediss, n¹9, pp.7-26.

Bertin-Mourot Bénédicte, 1997, «La participation des femmes à l'exercice du pouvoir dans les grandes entreprises : 4 études de cas », Les Cahiers du Mage, n ${ }^{\circ}$, pp.37-54.

CGE (Conférence des Grandes Ecoles, 2004, 2005, 2006, 2007, 2008, Résultats de l'enquête sur l'insertion des jeunes diplômés, Rapport.

CNISF, 2007, Rapport de la 18e enquête auprès des ingénieurs diplômés. Rapport.

Dubar Claude, 2001, «La construction de 1'insertion professionnelle», Education et Sociétés, n¹, pp.23-36.

Duru-Bellat Marie, 2004, L'école des filles. Quelle formation pour quels rôles sociaux? Paris, Harmattan, $2^{\mathrm{e}}$ éd.

Epiphane Dominique et Martinelli Daniel, 1997, «L'insertion professionnelle des jeunes femmes diplômées. En sortant des écoles: Qu'ont-elles rencontré? », Etude, n 70, pp.113-123.

Epiphane Dominique, 2006, «Les femmes et les sciences font-elles bon ménage? » in E., Flahault, L'insertion professionnelle des femmes. Entre contraintes et stratégies d'adaptation (pp.113-128). Paris, Des Sociétés.

Evetts Julia, 1998, "Managing the technologiy but not the organization: women and career in engineering », Women in Management Review, n $13(8)$, pp.283-290.

GEF (Grandes Ecoles au Féminin), 2007, «Parcours professionnels des hommes et des femmes diplômé(e)s de grandes écoles», Communiqué de presse.

Laufer Jacqueline, 1982, La féminité neutralisée? Les femmes cadres dans l'entreprise. Paris, Flammarion.

Laufer Jacqueline et Pochic Sophie, 2004, «Carrières au féminin et au masculin. Les nouvelles règles du jeu » in A., Karavar, A., Rouban L., Les cadres au travail (pp.147-169), Paris, La Découverte. 
Laufer Jacqueline, 2005, «La construction du plafond de verre: le cas des femmes cadres à potentiel », Travail et Emploi, $n^{\circ} 102$, pp.31-45.

Le Pellec Loïc et Roux Sébastien, 2001, «Les salaires des ingénieurs diplômés en 2000 », INSEE Première, $\mathrm{n}^{\circ} 812$.

Marry Catherine, 1995, «Polytechniciennes=polytechniciens ? », Les cahiers du Mage, n³-4, pp.7383.

Marry Catherine, 2004, Les femmes ingénieurs. Une révolution respectueuse, Paris, Belin.

Myrand Marie-Eve, 2008, Les femmes en sciences et génie: Les différents facteurs influençant leur sous-représentativité an milieu scolaire et professionnel. Essai, Université Laval, Québec.

Peslouan De Genviève, 1974, Qui sont les femmes ingénieurs en France?, Paris, PUF.

Phipps Alison, 2002, «Engineering women: The gendering of professional identities », The International Journal of Engineering Education, $n^{\circ} 18(4)$, pp.409-414.

Roberts Pam et Ayre Mary, 2002, «Did she jump or was she pushed? A study of women's retention in the engineering workforce », The International Journal of Engineering Education, $n^{\circ} 18(4)$, pp.415421.

Stevanovic Biljana, 2006, La mixité dans les écoles d'ingénieurs. Le cas de l'ex-Ecole Polytechnique Féminine, Paris, Harmattan.

Van Nieuwenhuyse Hélène, Descarries Francine et Deschenes Claire, 2003, « Mères et ingénieurs à la fois...» in C., Solar, L., Lafortune, Femmes et maths, sciences et technos (pp.147-162), Montréal, Presses de l'Université du Québec.

Verdier Eric, 1996, «L'insertion des jeunes « à la française » : vers un ajustement structurel », Travail et Emploi, $\mathrm{n}^{\circ} 69$, pp.37-59.

Vincent Bruno, 2010, Du plafond de verre aux plafonds des verres: l'inégal accès des femmes et des hommes aux emplois de direction générale des grandes collectivités territoriales, Rapport, ENSAE.

Women's Forum, 2008, Portrait de femmes ingénieurs, Rapport.

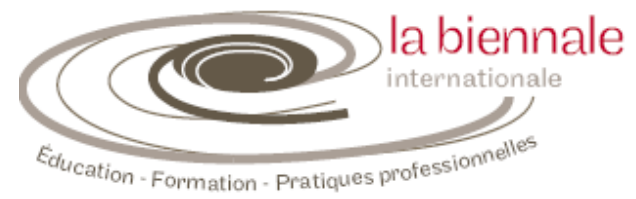

